\title{
Describing psychological and behavioural problems in Omani young people: Reliability of the self-reported Strength and Difficulties Questionnaire (SDQ) in Oman.
}

\author{
Al-Mukhaini ZA, Bekker HL, Cottrell D \\ Leeds Institute of Health Sciences, School of Medicine, University of Leeds, UK
}

\begin{abstract}
Aim: The aim of this study is to further assess the reliability of the Strength and Difficulties Questionnaire (SDQ) when completed by school-age children in Oman. The SDQ is an essential tool for screening psychological and behavioural problems in school children but has only been psychometrically tested once in Omani culture without checking the reliability of the impact subscale.

Method: A cross sectional study. Children aged 11-16 from one private and four public schools in two areas of Oman (Muscat; Buraimi) completed the SDQ. Cronbachs alpha and personproduct correlations were used to assess internal, and test-re-rest reliability.

Results: 377/422 children completed the SDQ, 138 completed the re-test. The Total Difficulties (T-SDQ) score was normally distributed with $78 \%$ scoring normal, $13 \%$ borderline and $9 \%$ clinical significant scores. Girls' scores were significantly higher than boys in T-SDQ and in emotional and prosocial subscales. Internal reliability for the T-SDQ was (Cronbach alpha $=0.72$; Person Product-moment coefficient $r=0.71 ; p<0.001$ ), the impact subscale $=0.68$ and varied between 0.26 and 0.60 for the other subscales.

Conclusion: The self-reported SDQ using T-SDQ score is a reliable screening tool for psychological and behavioural problems for Omani children. The T-SDQ can be disseminated within private and public-school systems. Having a reliable screening tool for identifying children with psychological and behavioural problems is a significant step to improving mental health pathways of care and outcomes in Oman.
\end{abstract}

Keywords: SDQ self-reported version, Reliability, Behavioural and psychological problems, Oman, children, Young people.

\section{Introduction}

Essential to the early and effective treatment of mental health problems in children is their identification. The Strength and Difficulties Questionnaire (SDQ) is an established measure used to screen for psychological and behavioural problems in children aged between 4 and 17 years old [1,2]. Self (child) and proxy (parent, teacher) completion versions have been used in community (e.g. school) and health care (e.g. hospital) settings. The SDQ consists of 25 items divided into five subscales: Conduct Problems, Hyperactivity Problems, Emotional Symptoms, Peer Problems and Pro-social Behaviour [3-5]. Both the total score excepting the pro-social behaviour items, and sub-scale totals are used to screen children, and assess the impact of treatment. The impact subscale supplement is optionally available for use. It is useful for clinicians and researchers as the respondent would put his/her perception if there is any impact on his/her life at home, school, friend relationship or leisure [1].

The SDQ has been translated into over 80 languages (www. sdq.sdqinfo.com), and psychometric testing indicates it is a reliable screening tool in different cultures [1,6-12]. The selfreport version of the SDQ has been translated into Arabic [1] and tested in Yemeni and Palestinian populations $[6,13,14]$. The SDQ-Arabic has been applied and psychometrically tested once in two large city populations in Oman [15]. In view of shortage in the number of mental health professionals in Oman [16], having a simple and short screening tool will be very useful for the mental health services in the country.

The aim of this study is to assess the reliability of the SDQ with the supplemented impact subscale when completed by schoolage children from a port and rural location in Oman, and to make a preliminary assessment of the level of psychological problems in Omani school children. Before the SDQ can be adopted as a general screening tool for psychological problems in Oman, its reliability needs to be assessed. Reliability is the internal consistency of a psychometric scale and its freedom from random error [17,18]. Reliability is defined as the "quantifying precision of a quantitative test" [17]. A reliable scale needs to be reproducible and often assessed by test-re-test as well as the inter-rater reliability [17-19]. 
Citation: Mukhaini AZ, Bekker HL, Cottrell D. Describing psychological and behavioural problems in Omani young people: Reliability of the self-reported Strength and Difficulties Questionnaire (SQD) in Oman. J Child Adolesc Health. 2018;2(2):19-44.

\section{Methods}

\section{Design and Sample}

A cross sectional study employing questionnaire methods in five school across two areas of Oman, Muscat (capital city) and Buraimi (border town and rural). Five schools were selected purposefully to ensure a diverse group of pupils: three Muscat schools - a private school of mixed sex, and two single-sex community schools (one for boys and for girls); two Buraimi schools - single-sex community schools (one for girls and one for boys). Schoolchildren aged 11-16 years were eligible to participate $(\mathrm{n}=442)$.

\section{Questionnaire}

The Arabic version of the SDQ self-report (child) with Impact Supplement was employed. The subscales scores for Emotional Symptoms (ES), Conduct Problems (CP), Hyperactivity (H), Peer Problems (PP) and ProSocial (PS) are rated 0-10. The total difficulty score (T-SDQ) is calculated by adding up the scores from all items within each sub-scale, excepting those in the pro-social sub-scale, i.e., a range from 0 to 40 . Higher scores indicate greater levels of difficulty; $0-15$ is indicative of a normal range, 16-19 is indicative of a borderline clinical indicator, and $20+$ as clinically significant levels [1]. The impact supplement subscale consists of five questions reflecting the child's perception that the condition has an impact on her/his life at home or school or leisure or peer-relationship. The impact score provides researchers and clinicians with insight into how the child's problems interfere with everyday activities and lives. Each item within the SDQ is rated as true (score=2), somewhat true $($ score $=1)$ or not true $($ score $=0)$.

\section{Procedure}

Ethical approval was granted by the Ministry of Health in Oman. Letters of study participation send to headmasters of all the five schools. and face-to-face meetings between ZA-M and the headmasters; all consented to participate. ZA-M worked with two teachers (ZA and SG) to distribute questionnaires across grade 6-10 classes (age 11-17) in all five schools. Pupils were given a description of the study and asked to complete the SDQ fully at two time-points (a month apart). Participants were informed their results would be treated in confidence by ZA-M and were not associated with their school marks and/or progress. In the private school, questionnaires were distributed by the teachers and the researcher and the researcher was able to address pupil queries; in the public schools, the teachers distributed the questionnaires with guidance from ZA-M on the study purpose and SDQ completion.

\section{Analysis}

Data were managed using SPSS. Analyses were carried out to describe the sample and assess the reliability of the SDQ scores using Cronbach's alpha coefficient for internal consistency [16], i.e., scores greater than 0.70 indicative of relaibility [17]. The test-re-test reliability analyses of the total difficulties SDQ scores was assessed using the Person Product-moment Correlation.

\section{Results}

Questionnaires were completed by 442 school children across five different schools in Oman (52 from Buraimi and 390 from Muscat); 201 pupils from the private schools and 241 from the public schools. Children were excluded from the analyses for those not completing the questions necessary to calculate the total difficulties (T-SDQ). There was no difference between those completed the SDQ $(n=377)$ and those with missed data $(n=65)$ in terms of age, sex and schools (Table 1). For the testretest reliability analysis, a sample of 138 children completed the self-report SDQ again, four weeks later.

The T-SDQ scores were distributed normally across the sample (Figure 1) and supported by similarities in the mean (11.9 \pm $5.3)$ and median (12.00) average scores, and 5\% trimmed mean (11.7) analysis; $\mathrm{t}(376)=0.673, \mathrm{P}=0.50,95 \%$ CI $(-.35,0.72)$. Most (78\%) pupils fell within the normal range for the SDQ; $14 \%$ were within the borderline category and $9 \%$ clinically significant range (Table 2). The SDQ subscale scores and impact score illustrate all scores were skewed towards the normal range. The mean impact score was $1.26 \pm 1.92$.

There was no significant difference in T-SDQ scores between private and public schools (private mean $11.5 \pm 5.4$; public mean $12.2 \pm 5.2 ; \mathrm{t}(170)=-1.58, \mathrm{P}=0.116,95 \% \mathrm{CI}(-1.46,0.16))$. Girls scored higher than boys (boys mean $11.2 \pm 5.2$; girls mean $12.5 \pm 5.3 ; \mathrm{t}(375)=-2.37, \mathrm{p}<0.05,95 \% \mathrm{CI}(-2.35,-0.22))$. There was no difference between boys and girls in conduct problem, hyperactivity and peer problem subscales' scores; girls scored higher than boys on the emotional symptoms, pro-social subscales and impact score (Table 3 ).

Cronbach's alpha (T-SDQ) was 0.72 , indicating good internal consistency; values for the subscales were modest-to-poor: ES (0.60), CP (0.45), Hyperactivity (0.47), PP (0.26), and PS (0.42). For Impact score the Cronbach alpha was 0.68 . The test-retest correlation was significantly high (Person Product-moment coefficient $\mathrm{r}=0.71, \mathrm{p}<0.001$ ). Cronbach's alpha (T-SDQ) is like some studies in literature $[10,21,22]$. Table (4) compares the Cronbach's alpha between present study and literature.

Compared with children in other countries, Omani children's

Table 1. Description of 442 completed questionnaires.

\begin{tabular}{|c|c|c|c|c|c|c|}
\hline \multirow{2}{*}{ Case Types } & \multicolumn{2}{|c|}{ Age in years } & \multicolumn{2}{|c|}{ Sex Number (\%) } & \multicolumn{2}{|c|}{$\begin{array}{c}\text { Number of Students defined by school } \\
\text { number (\%) }\end{array}$} \\
\hline & Mean $\pm(S D)$ & $95 \% \mathrm{Cl}$ & Male & Female & Public school & Private school \\
\hline With complete data $(n=377)$ & $13.32 \pm(1.46)$ & $13.17-13.47$ & $174(46.2)$ & $203(53.8)$ & $204(54.1)$ & $173(45.9)$ \\
\hline With missed data $(n=65)$ & $13.41 \pm(1.51)$ & 13.03-13.78 & $22(33.8)$ & $43(66.2)$ & $37(56.9)$ & $28(43.1)$ \\
\hline
\end{tabular}


Table 2. Distribution of total difficulties score groups in both sexes $(n=377)$.

\begin{tabular}{|c|c|c|c|c|c|c|}
\hline \multirow{2}{*}{ T-SDQ (groups) } & \multicolumn{2}{|c|}{ Male } & \multicolumn{2}{|c|}{ Female } & \multicolumn{2}{|c|}{ Total } \\
\hline & Number & $(\%)$ & Number & $(\%)$ & Number & $(\%)$ \\
\hline Normal (Scores $\leq 15$ ) & 141 & 48 & 152 & 52 & 293 & $78 \%$ \\
\hline Borderline (Scores 16-19) & 22 & 43 & 29 & 57 & 51 & $13 \%$ \\
\hline Clinically Significant (Scores $\geq 20$ ) & 11 & 33 & 22 & 67 & 33 & $9 \%$ \\
\hline Total & 174 & 46 & 203 & 54 & 377 & 100 \\
\hline
\end{tabular}

Table 3. Mean scores of all five subscales and impact subscale and Mann-Whitney U test findings of subscales comparing female and male scores $(n=377)$.

\begin{tabular}{|c|c|c|c|c|c|}
\hline \multirow{2}{*}{ Subscales } & \multicolumn{3}{|c|}{ Mean score \pm (S.D) } & \multirow{2}{*}{ Z-value } & \multirow{2}{*}{ P-value } \\
\cline { 2 - 5 } & Both sexes & Males & $3.97 \pm(2.25)$ & -5.09 & $<0.001$ \\
\hline Emotional symptoms & $3.46 \pm 2.22$ & $2.82 \pm(2.01)$ & $2.99 \pm(1.76)$ & -1.94 & NS \\
\hline Peer Problems & $2.85 \pm 1.70$ & $2.64 \pm(1.59)$ & $2.34 \pm(1.77)$ & -0.791 & NS \\
\hline Conduct Problems & $2.36 \pm 1.72$ & $2.38 \pm(1.64)$ & $3.36 \pm(1.89)$ & -0.088 & NS \\
\hline Hyperactivity & $3.35 \pm 1.88$ & $3.35 \pm(1.88)$ & $8.04 \pm(1.65)$ & -2.37 & $<0.018$ \\
\hline Pro-social & $7.91 \pm 1.63$ & $7.73 \pm(1.59)$ & $1.62 \pm 2.16$ & -3.86 & $<0.001$ \\
\hline Impact Score & $1.26 \pm 1.92$ & $0.82 \pm 1.46$ & & & \\
\hline
\end{tabular}

Table 4. Comparison of Cronbach's alpha of study scores $(n=377)$ with some other studies in literature.

\begin{tabular}{|c|c|c|c|c|c|c|c|c|c|c|}
\hline \multirow{2}{*}{ Year \& Author } & \multirow{2}{*}{$\begin{array}{l}\text { Sample } \\
\text { size }\end{array}$} & \multirow{2}{*}{ Sample population } & \multirow{2}{*}{ Country or Ethnicity } & \multicolumn{7}{|c|}{ Internal consistency (Cronbach Alpha) } \\
\hline & & & & T-SDQ & ES & $\mathrm{CP}$ & Hyperactivity & PP & PS & Impact \\
\hline Goodman [1] & 199 & Community & UK & $\begin{array}{c}0.61 \\
\text { to } \\
0.82\end{array}$ & - & - & - & - & - & - \\
\hline Goodman, et al. [2] & 199 & $\begin{array}{l}\text { Community and } \\
\text { clinic }\end{array}$ & $\begin{array}{c}\text { UK } \\
\text { Caucasian }\end{array}$ & 0.82 & 0.8 & 0.7 & 0.59 & 0.7 & 0.6 & - \\
\hline Koskelainen, et al. [10] & 735 & Community & Finland & 0.71 & 0.7 & 0.7 & 0.57 & 0.6 & 0.7 & - \\
\hline Thabet, et al. [13] & 322 & Community & Palestine & - & 0.5 & 0.3 & 0.55 & 0.2 & 0.7 & - \\
\hline Emam, et al. [15] & $634-815$ & Community & Oman & 0.76 & 0.6 & 0.5 & 0.51 & 0.4 & 0.5 & - \\
\hline Van Widenfelt, et al. [20] & 1288 & Community & Netherlands & 0.7 & 0.6 & 0.5 & 0.66 & 0.4 & 0.6 & - \\
\hline $\begin{array}{l}\text { Mansbach-Kleinfeld, et } \\
\text { al. [21] }\end{array}$ & 611 & Community & $\begin{array}{l}\text { Isreal } \\
\text { Hebrew }\end{array}$ & 0.72 & 0.6 & 0.5 & 0.71 & 0.2 & 0.6 & - \\
\hline Muris, et al. [23] & 439 & Community & Netherlands & 0.76 & 0.6 & 0.5 & 0.68 & 0.4 & 0.6 & - \\
\hline Giannakopoulos, et al. [26] & 1194 & Community & Greek & 0.77 & 0.73 & 0.6 & 0.63 & 0.6 & 0.6 & - \\
\hline Du, et al. [27] & 690 & Community & China & 0.57 & 0.6 & 0.3 & 0.64 & 0.3 & 0.7 & - \\
\hline Ortuno-Sierra, at al. [28] & 1474 & Community & Spain & 0.75 & 0.71 & 0.6 & 0.68 & 0.6 & 0.6 & - \\
\hline Present study & 377 & Community & Oman & 0.72 & 0.6 & 0.5 & 0.47 & 0.3 & 0.4 & 0.68 \\
\hline
\end{tabular}

total difficulties score is similar to Australian children [23] but higher than children in other cultures [10,22-24]. There were some consistent differences reported between male and female responses on subscales across countries, such as girls scoring high on the Emotional symptoms score [10,24,25-28] and Prosocial score [25,28]. The same findings reported in this study.

\section{Discussion}

This study is the first to test the reliability of the supplemented SDQ in a population of Omani schoolchildren from both private (affluent) school and public (general) school. No significant difference in T-SDQ mean score between private and public schoolchildren might indicate that the socio-economic-class has no influence on Omani young people psychological health. Although the T-SDQ has been tested and used in several different English [2-5] and non-English, speaking countries [6-15] there are few examples of it being used in Arabic populations and culture $[6,14,16]$. The findings indicate the T-SDQ is a reliable screening tool for psychological and behavioural problems in Omani children aged 11-16 years. Having a reliable screening tool for identifying children with psychological and behavioural problems is a first step to improving mental health pathways of care and outcomes. This study illustrated the acceptability of disseminating and utilizing the T-SDQ within private and publicschool systems and suggests a model of collaboration between school and health care systems when implemented in practice. The impact subscale showed reasonably good Cronbach alpha coefficient (0.68).

The findings illustrated a normal distribution of responses, and discrimination between normal, borderline and clinical significant responses in accordance with external indicators of mental health prevalence in populations [1]. However, the study did not explore the validity of the items and questionnaire, and it may be Omani pupil's understanding and/or interpretation of items differ from children in other cultures. This may have implications for the sensitivity and specificity of the test, and implications for service provision. For example, it is unclear if sex differences in responses represent a difference in illness prevalence between girls and boys, or a difference in reporting. It is recognized that girls develop and articulate emotions and social relationships before boys [29], suggesting the T-SDQ may be misclassifying boys as normal or borderline. Future research and audit would be able to address this type of issues and adapt classification algorithms accordingly. 
Citation: Mukhaini AZ, Bekker HL, Cottrell D. Describing psychological and behavioural problems in Omani young people: Reliability of the self-reported Strength and Difficulties Questionnaire (SQD) in Oman. J Child Adolesc Health. 2018;2(2):19-44.



Figure 1. Histogram illustrating distribution of $S D Q$ responses by participant.

\section{Conclusion}

The SDQ looks at strength as well as difficulties and it is more acceptable to respondents and gives richer information. It is short and easy to administer (one side of A4 paper if SDQ alone or two sided A4 paper if the SDQ with impact subscale is used) which makes it a quick, useful screening tool for both clinician and researcher

\section{Acknowledgments}

I would like to thank all teachers, students and head teachers for their cooperation without which this study would not be possible. I declare that there is no conflict of interest on this project. I would like to thank government of The Sultanate of Oman that supported me to do this study by sabbatical leave

\section{References}

1. Goodman R. The strengths and difficulties questionnaire: A research note. J Child Psychol Psychiatry. 1997;38(5):581-6.

2. Goodman R, Ford T, Simmons H, Gatward R, Meltzer H. (2000). Using the Strengths and Difficulties Questionnaire (SDQ) to screen for child psychiatric disorders in a community sample. British Journal of Psychiatry. 2000 177: 534-539.

3. Goodman R. The extended version of the strengths and difficulties questionnaire as a guide to child psychiatric caseness and consequent burden. J Child Psychol Psychiatry. 1999;40(5):791-9.

4. Goodman R, Ford T, Simmons H, et al. Using the strengths and difficulties questionnaire to screen for child psychiatric disorders in a community sample. $\mathrm{Br} \mathrm{J}$ Psychiatry. 2000;177(6):534-9.

5. Goodman R, Scott S. Comparing the strengths and difficulties questionnaire and the child behavior checklist: Is small beautiful? J Abnorm Child Psychol. 1999;27(1):17-24.

6. Almaqrami MH, Shuwail AY. Validity of the self-report version of the strengths and difficulties questionnaire in Yemen. Saudi Med J. 2004;25(5):592-601.

7. Fleitlich B, Goodman R. Social factors associated with child mental health problems in Brazil: Cross sectional survey.
Bmj. 2001;32(3):599-600

8. Smedje H, Broman JE, Hetta J, et al. Psychometric properties of a Swedish version of the "Strengths and Difficulties Questionnaire". Eur Child Adolesc Psychiatry.1999;8(2):63-70.

9. Klasen H, Woerner W, Wolke D, et al. Comparing the German versions of the strengths and difficulties questionnaire and the child behavior checklist. Eur Child Adolesc Psychiatry. 2000;9(4):271-6.

10. Koskelainen M, Sourander A, Kaljonen A. The strengths and difficulties questionnaire among finnish school-aged children and adolescents. Eur Child Adolesc Psychiatry. 2000;9(4):277-84.

11. Mathai J,Anderson P, Bourne A. The strengths and difficulties questionnaire as a screening measure prior to admission to a Child and Adolescent Mental Health Service (CAMHS). Australia J Advance Mental Health. 2002;1(3):235-46.

12. Mullick MS, Goodman R. Questionnaire screening for mental health problems in Bangladeshi children: a preliminary study. Soc Psychiatry Psychiatr Epidemiol. 2001;36(2):94-9.

13. Thabet AA, Stretch D, Vostanis P. Child mental health problems in Arab children: Application of the strengths and difficulties questionnaire. Int $\mathrm{J}$ Soc Psychiatry. 2000;46(4):266-80.

14. Alyahri A, Goodman R. Validation of the Arabic strength and difficulties questionnaire and the Development of Well Being Assessment. East Mediterr Health J. 2006;12(2):138-46.

15. Emam MM, Kazem AM, Al-Zubaidy AS. Emotional and behavioural difficulties among middle school students in Oman: an examination of prevalence rate and gender differences. Emotion Behav Difficult. 2016;2;21(3):314-28.

16. Al-Sinawi H, Al Adawi S. Psychiatry in the Sultanate of Oman. Bulletin of the Board of International Affairs of the Royal College of Psychiatrists. 2006; 3(4).

17. Daley LE, Bourke GJ. Interpretation and uses of medical statistics. Blackwell, Oxford, UK. 2003. 
18. Bowling A. Research methods in health: Investigating health and health services. Open University Press, Buckingham, Philadelphia. 2000.

19. Pallant J. SPSS Survival Manual. Open University Press, Buckingham, Philadelphia. 2002.

20. Van Widenfelt BM, Goedhart AW, Treffers PD, et al. Dutch version of the strengths and difficulties questionnaire. Eur Child Adolesc Psychiatry. 2003;12(6):281-9.

21. Mansbach-Kleinfeld I, Apter A, Farbstein I, et al. A population-based psychometric validation study of the strengths and difficulties questionnaire: Hebrew version. Frontier Psychiat. 2010;1:151.

22. Hawes DJ, Dadds MR. Australian data and psychometric properties of the strengths and difficulties questionnaire. Aust N Z J Psychiatry. 2004;38(8):644-51.

23. Muris P, Meesters C, Eijkelenboom A, et al. The self-report version of the strengths and difficulties questionnaire: Its psychometric properties in 8-to 13-year-old non-clinical children. Br J Clin Psychol. 2004;43(4):437-48.

24. Van Roy B, Veenstra M, Clench-Aas J. Construct validity of the five-factor strengths and difficulties questionnaire in pre-early and late adolescence. J Child Psychol Psychiatry. 2008;49(12):1304-12.

25. Bøe T, Hysing M, Skogen JC, et al. The strengths and difficulties questionnaire: Factor structure and gender equivalence in Norwegian adolescents. PLOS One. 2016;11(5):e0152202.

26. Giannakopoulos G, Tzavara C, Dimitrakaki C, et al. The factor structure of the strengths and difficulties questionnaire in Greek adolescents. Ann Gen Psychiatry. 2009;8(1):20.

27. Du Y, Kou J, Coghill D. The validity, reliability and normative scores of the parent, teacher and self-report versions of the strengths and difficulties questionnaire in China. Child Adolesc Psy Mental Health. 2008;2(1):8.

28. Ortuno-Sierra J, Fonseca-Pedrero E, Paino M, i Riba SS, Muniz J. Screening mental health problems during adolescence: Psychometric properties of the Spanish version of the Strengths and Difficulties Questionnaire. Journal of Adolescence. 2015 Jan 1;38:49-56.

29. Chaplin TM, Aldao A. Gender differences in emotion expression in children: Ameta-analytic review. Psychological bulletin. 2013;139(4):735

\section{*Correspondence to:}

Al-Mukhaini ZA

Research Fellow - Leeds Institute of Health Sciences, School of Medicine, University of Leeds, UK. e.mail : zalmukhini@yahoo.com 